\title{
Direct Measurement of Kinetic Parameters of ABCG2-Dependent Transport of Natural Flavonoids Using a Fluorogenic Substrate
}

\author{
Michal Rozanski, @Maciej Studzian, and 니k \\ Laboratory of Transcriptional Regulation, Institute of Medical Biology, Polish Academy of Sciences, Lodz, Poland (M.R., L.P.); \\ Department of Molecular Biophysics, Faculty of Biology and Environmental Protection, University of Lodz, Lodz, Poland (M.S., \\ L.P.); and Department of Molecular Biology of Cancer, Medical University of Lodz, Lodz, Poland (M.R.)
}

Received July 4, 2019; accepted August 28, 2019

\begin{abstract}
Flavonoids are an important part of the human diet since plantderived polyphenols and the mechanisms governing their pharmacokinetics are important both due to their own nutriceutical activity and the potential for food-drug interactions. A central determinant of absorption and distribution of flavonoids in the human body is the ATP-binding cassette transporter ABCG2, expressed in gut epithelium and other barrier tissues. While flavonoids were previously identified as substrates and/or inhibitors of this protein, precise enzyme kinetic calculations of affinity and activity parameters are rare due to the lack of suitable experimental models. We present a novel method that allows the direct measurement of kinetic constants for ABCG2-mediated cellular efflux of natural flavonoids thanks to the application of fluorogenic 2-aminoethyl diphenylborinate, which reacts with intracellular flavonoids forming a fluorescent, nonmembranepermeable conjugate, thus making it possible to measure the intracellular substrate concentration throughout the experiment. Our studies were performed in Madin-Darby canine kidney IIderived cell lines expressing human ABCG2 and involve substrate
\end{abstract}

efflux from whole, unmodified cells, precluding the need for plasma membrane vesicle preparation. We present methods for calculation of enzyme kinetic constants by measuring substrate concentration at efflux-influx equilibrium or during efflux from preloaded cells, and we obtained $K_{\mathrm{m}}$ values of $137 \mu \mathrm{M}$ for quercetin, $36 \mu \mathrm{M}$ for kaempferol, and $348 \mu \mathrm{M}$ for luteolin. Our method also allows direct verification of the transport inhibition mechanism and potentially the structure-activity relationship in substrates.

\section{SIGNIFICANCE STATEMENT}

The study presents the first direct calculation of kinetic constants for enzyme-mediated active transport of natural flavonoids in a whole-cell assay, using a fluorogenic compound to measure intracellular substrate concentrations at specific time points. It has implications for nutriceutical use of polyphenols, mechanisms of food-drug interactions, and studies on absorption/ distribution-determining membrane transporters, allowing a quantitative approach to pharmacokinetics of flavonoid transport across barrier tissues.

\section{Introduction}

Proteins of the ATP-binding cassette (ABC) superfamily are membrane efflux transporters, actively pumping out xenobiotics outside of the cell (Ford and Beis, 2019). One among the best known ABC proteins is ABCG2, also known as breast cancer resistance protein. ABCG2 is a so-called halftransporter, meaning that it is a homodimer of which each subunit (polypeptide chain) contains one nucleotide binding domain $(\mathrm{ABC})$ and a hexahelical transmembrane domain. The spatial structure of human ABCG2 was resolved recently via cryo-electron microscopy (Taylor et al., 2017). Physiologically, ABCG2 is expressed mainly in barrier tissues such as the intestine (Sawangrat et al., 2019), placenta (Mao, 2008; Szilagyi et al., 2019), or blood-brain barrier (Iorio et al., 2016) and significantly affects the pharmacokinetics of many drugs (Shugarts and Benet, 2009; Robey et al., 2011).

This work was supported in part by the Polish National Science Centre [Project No. 2015/19/B/NZ7/03856].

https://doi.org/10.1124/jpet.119.261347.
While precise biochemical knowledge about $\mathrm{ABC}$ transporters is extremely helpful in predicting their physiologic relevance, e.g., for drug-drug interactions (Giacomini et al., 2010), determining basic kinetic constants for active membrane transporters has always been a demanding task. Direct measurements of the concentration of complex organic substrates (such as anticancer drugs) are dependent on the availability of radioactively labeled compounds or require sophisticated and artifact-prone methods such as liquid chromatography and mass spectrometry (Xie et al., 2015; Li et al., 2017). Therefore, researchers often use model substrates, which can be detected more easily using fluorimetric, colorimetric, or enzymatic assays. It is also common to measure the ATPase activity of the nucleotide binding domain instead of the transporter activity itself (Glavinas et al., 2007).

When values of enzyme kinetic constants are required for $\mathrm{ABC}$ proteins, three approaches are predominant in the literature. The first one involves measuring equilibrium concentrations of a substrate on the apical and basolateral sides of a cellular monolayer (Mukkavilli et al., 2017). This assay depends on membrane domain-specific expression of

ABBREVIATIONS: ABC, ATP-binding cassette; APB, 2-aminoethyl diphenylborinate; BSA, bovine serum albumin; DMEM, Dulbecco's modified Eagle's medium; HBSS, Hanks' balanced salt solution; LB, lysis buffer; MDCK, Madin-Darby canine kidney; WT, wild type. 
the protein of interest in polarized cell lines. The second approach depends on the use of isolated membrane vesicles containing transporter molecules (Pedersen et al., 2017). These methods often depend on heterologous overexpression of transporters, usually in insect cell lines. The third approach uses bioinformatic modeling for prediction of kinetic parameters for a new substrate (Gantner et al., 2017) or to build in silico transport models (Schutte et al., 2006).

In this article, we present the direct measurement of enzyme kinetic constants for ABCG2 with regard to flavonoids as natural substrates. They are ubiquitous polyphenolic plant secondary metabolites, abundant in diet and with many recognized bioactivities in the human body (Fraga et al., 2019). Many of them are known or predicted to be substrates of the ABCG2 transporter (An et al., 2011; Ge et al., 2016; Iriti et al., 2017; Sjöstedt et al., 2017; Xiang et al., 2018). Intestinal expression of ABCG2 defines it as one of the crucial determinants of absorption and distribution of dietary compounds, and also as the primary location of food-drug and drug-drug pharmacokinetic interactions. While facilitated flavonoid transport has been described for some mammalian transporters from the solute carrier superfamily, such as glucose transporters 1,2 , and 4 or organic anion transporter 1 , only $\mathrm{ABC}$ transporters (ABCG2 and ABCB1) have been demonstrated to catalyze active flavonoid efflux (Passamonti et al., 2009; Williamson et al., 2018).

In this study, we apply the fluorogenic probe 2-aminoethyl diphenylborinate (APB) in a dual role: 1) to fix flavonoids inside the cells, preventing their further transport; and 2) to measure their intracellular concentration directly by fluorimetry. The rationale of this method has been previously described by us (Sadowska-Bartosz et al., 2016; Studzian and Pulaski, 2017); here, we modify and optimize it to allow the direct derivation of kinetic constants of transport. This method requires a stringently defined cellular model; in our case, a commercially available Madin-Darby canine kidney (MDCK) II-derived cell line pair, which differs exclusively in the expression of human ABCG2.

\section{Materials and Methods}

Cell Culture. We used MDCKII-derived cell lines (Hera Biolabs, Lexington, KY), which have CRISPR/Cas9-mediated inactivation of canine $a b c g 2$ and $a b c b 1$ genes. The cell lines were MDCKII-BP-null [control line, designated as MDCK wild-type (WT)] and hBCRPMDCKII-BP-null (cell line stably transfected with the human $A B C G 2$ gene, designated as MDCK-G2) [https://www.herabiolabs.com/mdckbp-null-cells/]. Cell culture was performed under standard conditions $\left(37^{\circ} \mathrm{C} ; 5 \% \mathrm{CO}_{2}\right)$. Cells were maintained in Dulbecco's modified Eagle's medium (DMEM) (Life Technologies) supplemented with Glutamax and $10 \%$ FBS (PAN, Biotech), without antibiotics.

Stock Solutions and Buffers. 2-Aminoethyl diphenylborinate was purchased from Sigma, dissolved in DMSO to a concentration of $125 \mathrm{mg} / \mathrm{ml}$, and then stored at $-20^{\circ} \mathrm{C}$. Flavonoid stock solutions were prepared at a concentration of $25 \mathrm{mM}$ in DMSO and stored at $-20^{\circ} \mathrm{C}$. Working solutions were prepared in DMEM (Life Technologies), Hanks' balanced salt solution [(HBSS); Life Technologies], or PBS (Sigma-Aldrich). In some cases, bovine serum albumin [(BSA); SigmaAldrich] or fatty acid-free BSA (Sigma-Aldrich) was included in the solutions at a concentration of $2 \mathrm{mg} / \mathrm{ml}$. Lysis buffer (LB) was prepared by dissolving APB from the stock solution to a final concentration of $250 \mu \mathrm{g} / \mathrm{ml}$ and Triton X-100 to a final concentration of $0.35 \%(\mathrm{v} / \mathrm{v})$ in PBS.

Fluorescence Measurement. Twenty-four hours before the experiment, MDCKII cells were plated on a standard, black 96 -well plate at a density of 40,000 cells per well. Flavonoids were dissolved from the stock solution to a desired concentration in HBSS/BSA. Cell culture medium was removed and cells were washed briefly with PBS at room temperature, and then PBS was replaced with $100 \mu \mathrm{l}$ of flavonoid solution. From this point on, the whole experiment was conducted in a thermostated glove chamber (Whitley H35 Hypoxystation; Don Whitley Scientific) with controlled atmosphere $\left(37^{\circ} \mathrm{C}\right.$; $5 \% \mathrm{CO}_{2}$ ). Cells were incubated with flavonoids for 40 minutes (until equilibrium was reached). For equilibrium concentration measurement, flavonoid solutions were replaced with $100 \mu \mathrm{l}$ of PBS/APB. For kinetic (efflux) concentration measurement, flavonoid solutions were first replaced by $100 \mu \mathrm{l}$ of DMEM/BSA and subsequently (after specific time periods) by the same volume of PBS/APB. In both cases, cells were incubated with PBS/APB for 10 minutes and then washed once with PBS. PBS was carefully removed and cells were lysed with $100 \mu \mathrm{l}$ of LB. After 5-minute incubation at room temperature, the plate was placed in an EnVision 2103 Multimode Reader (PerkinElmer), shaken for 5 minutes, and fluorescence intensity was measured at $550 \mathrm{~nm}$ emission and $485 \mathrm{~nm}$ excitation wavelengths. Independently, the fluorescence of a dilution series of flavonoids in LB was measured at the same conditions.

Protein Assay. Bradford reagent was prepared by dissolving $50 \mathrm{mg}$ of Coomassie Brilliant Blue G250 in $25 \mathrm{ml}$ of methanol, and then adding $50 \mathrm{ml}$ of concentrated phosphoric (V) acid. Next, the solution was diluted with Milli-Q water up to $500 \mathrm{ml}$ and filtered. Bradford reagent was aliquoted into a clear 96 -well plate $(200 \mu \mathrm{l}$ per well). Then, $5 \mu \mathrm{l}$ of a cellular lysate sample or $5 \mu \mathrm{l}$ of lysis buffer (blank) was added to each well and thoroughly mixed. After 2 minutes of incubation at room temperature, the plate was placed in the EnVision 2103 reader, shaken for 1 minute, and absorbance was measured at 450 and $590 \mathrm{~nm}$. Protein concentration in a sample is directly proportional to the ratio of $590-450 \mathrm{~nm}$ absorbance (Ernst and Zor, 2010).

Flavonoid Concentration Calculation. To calculate the intracellular flavonoid concentration, a measure of total cell volume in the well is necessary. We found that while the volume of individual MDCKII cells can fluctuate depending on culture state and cell subline, the protein concentration in the cell content is constant. Using the Bradford protein assay and optical microscope with a Neubauer improved cell counting chamber, we determined that the ratio of the cell volume to the protein mass for both MDCKII sublines was $14.5 \mu \mathrm{l} / \mathrm{mg}$ (data not shown). Thus, the average intracellular concentration of flavonoids can be described with following equation:

$$
C_{\text {flavo in cells }}=\frac{C_{\text {flavo }}}{C_{\text {prot }} \times 14.5}
$$

where $C_{\text {flavo }}$ is the flavonoid concentration in a well after lysis (in micromolars), calculated from the fluorescence values and the calibration curve; $C_{\text {prot }}$ is the protein concentration in a well after lysis (in micrograms per milliliter); and 14.5 is the cell volume to the protein mass coefficient.

Measurement of the Passive Transport Constant. Passive transport of flavonoids through the plasma membrane can be described by first-order kinetics. To measure the kinetic constant $k$, MDCK-WT cells were incubated to equilibrium with flavonoids and their intracellular (initial) concentration was measured. These cells contain no known active flavonoid transporters, since the expression of Abcg2 and Abcb1 has been genetically disrupted, and the known facilitated flavonoid transporters from the solute carrier superfamily that are expressed in these cells, such as Glut2 and Oat1, are expected to have negligible contribution to the passive flavonoid flux in view of the high unfacilitated (flip-flop) transport rate for the investigated flavonoids (aglycons, which are substantially less polar than flavonoid glycosides). Subsequently, a kinetic measurement was performed as described previously by replacing the HBSS/BSA/flavonoid solution with DMEM/BSA and measuring the intracellular concentration at 
specific time points. A first-order substrate decay curve, described by the following equation, was fitted to experimental points using the least-squares method:

$$
C_{\mathrm{WT}}=C_{0} \cdot e^{-k \cdot t}
$$

where $C_{\mathrm{WT}}$ is the intracellular flavonoid concentration in MDCK-WT cells (in micromolars); $C_{0}$ is the initial concentration (in micromolars); $k$ is the first-order kinetic constant (in liters per second); and $t$ is the time (in seconds).

Calculation of the $K_{m}$ and $V_{\max }$ Values for ABCG2 from Equilibrium Data. The availability of two cell lines that are identical in everything except ABCG2 expression makes it possible to derive the velocity of ABCG2-mediated flavonoid transport from intracellular concentration values at equilibrium. The derivation is based on the following premises:

1. Passive diffusion through membranes is a first-order process driven by the concentration of flavonoid on each side of the membrane.

2. At equilibrium, the net flavonoid flux through the membrane is equal to zero (the influx and efflux velocities are equal). In MDCK-WT cells, the equilibrium can be described by the equation $V_{\text {WTpas.in }}=V_{\text {WTpas.eff }}$, where $V_{\text {WTpas.in }}$ is the velocity of passive influx and $V_{\text {WTpas.out }}$ is the velocity of passive efflux. In MDCK-G2 cells, where an active efflux component appears alongside passive efflux, this equation takes the following form: $V_{\mathrm{G} 2 \text { pas.in }}=V_{\mathrm{G} 2 \text { pas.eff }}+V_{\mathrm{ABCG} 2}$, where $V_{\mathrm{G} 2 \text { pas.in }}$ is the velocity of passive influx; $V_{\mathrm{G} 2 p a s . o u t}$ is the velocity of passive efflux; and $V_{\mathrm{ABCG} 2}$ is the velocity of $\mathrm{ABCG} 2$-mediated active transport.

3. The presence of active efflux shifts the equilibrium such that at the same extracellular concentration of flavonoid (leading to the same influx velocity) a lower intracellular one is reached; thus, under these conditions the passive efflux velocity from the MDCK-G2 cells decreases (since it is driven by a lower concentration of flavonoid).

At equal extracellular concentrations of flavonoid, the passive influx velocities are equal in both cell lines. Because of the huge difference between extracellular and intracellular volume, transport processes leading to equilibrium will not lead to a detectable change in extracellular concentration of flavonoid in a cell culture microplate well. Therefore, at equilibrium the total efflux velocities (in MDCKWT cells it is assumed to be composed only of the passive component due to the lack of expression of any known active flavonoid transporters; in MDCK-G2 cells it comprises both passive and ABCG2derived active components) are also equal:

$$
V_{\text {WTpas.eff }}=V_{\mathrm{G} 2 \text { pas.eff }}+V_{\mathrm{ABCG} 2}
$$

where the velocity of the ABCG2-mediated transport is thus equal to the difference between the equilibrium velocities of the passive efflux in MDCK-WT and MDCK-G2 cells. We can use the previously derived passive transport constant to express the passive efflux velocities as functions of intracellular flavonoid concentration:

$$
\begin{gathered}
V_{\mathrm{ABCG} 2}=V_{\mathrm{WT} \text { pas.eff }}-V_{\mathrm{G} 2 \text {.pas.eff }} \\
V_{\mathrm{ABCG} 2}=k \cdot\left(C_{\mathrm{WT}}-C_{\mathrm{G} 2}\right)
\end{gathered}
$$

Therefore, by measuring intracellular flavonoid concentrations in both cell lines at a series of equal extracellular concentrations, we acquire a data set of substrate concentration $\left(C_{\mathrm{G} 2}\right)$ and enzyme velocity $\left(V_{\mathrm{ABCG} 2}\right)$, allowing us to plot a Michaelis-Menten curve and fit it to experimental points using the least-squares method:

$$
V_{\mathrm{ABCG} 2}=\frac{V_{\mathrm{max}} \cdot C_{\mathrm{G} 2}}{K_{\mathrm{m}}+C_{\mathrm{G} 2}}
$$

where $V_{\mathrm{ABCG} 2}$ is the ABCG2-mediated efflux velocity (in micromolars per second); $V_{\max }$ is the maximal velocity of ABCG2-mediated efflux (in micromolars per second); $K_{\mathrm{m}}$ is the Michaelis constant (in micromolars); and $C_{\mathrm{G} 2}$ is the intracellular flavonoid concentration in MDCK-G2 cells (in micromolars).

Calculation of the $K_{m}$ and $V_{\max }$ Values for ABCG2 from Kinetic Data. Kinetic (efflux) experiments were performed as described previously both for MDCK-WT and MDCK-G2 cells, measuring intracellular flavonoid concentration at specific time points after removal of extracellular flavonoid solution. A full analytical description of the flavonoid efflux kinetics in MDCK-G2 cells (where transport is both passive and active) would require an inexact numerical solution to a complex differential equation (resulting in a kinetic equation). Instead, we performed statistical analysis by nonlinear regression of the velocity equation (linking the transport velocity to the substrate concentration). The MDCK-G2 efflux kinetics is a combination of first-order passive efflux and Michaelis-Menten-based active efflux. Therefore, for each time point, the expected concentration can be calculated recurrently from data for the previous time point, thus simulating the entire combined curve. This curve then undergoes nonlinear regression using a four-parameter equation:

$$
\begin{gathered}
C_{t}=C_{t-1}-\left[V_{\mathrm{ABCG} 2}+V_{\text {pas.eff }}\right] \cdot \Delta t \\
C_{t}=C_{t-1}-\left[\left(\frac{V_{\max } \cdot C_{t-1}}{K_{\mathrm{m}}+C_{t-1}}\right)+k \cdot C_{t-1}\right] \cdot \Delta t
\end{gathered}
$$

where $C_{t}$ is the flavonoid intracellular concentration at the current simulated time point (in micromolars); $C_{t-1}$ is the flavonoid intracellular concentration at the previous simulated time point (in micromolars); $V_{\max }$ is the maximal velocity of ABCG2-mediated efflux (in micromolars per second); $K_{\mathrm{m}}$ is the Michaelis constant (in micromolars); $k$ is the passive efflux constant (in liters per second); $\Delta t$ is the time interval of simulation (in seconds); and $C_{0}$ is the initial intracellular flavonoid concentration (first point of simulation).

This allows the approximate calculation of enzyme constants (equation parameters) without the need to solve the differential velocity equation. Since multiple-parameter regression can lead to relatively good correlation coefficients for a wide range of parameter values, we had to set knowledge-based limits in the mathematical procedure of parameter calculation. The passive efflux constant was known (calculated previously from experiments on MDCK-WT cells); therefore, we tested five approaches to boundary conditions in calculating the $K_{\mathrm{m}}$ and $V_{\max }$ values (implemented within the leastsquares regression algorithm): 1) fixing either of the parameters to the equilibrium experiment-derived value (fixed $K_{\mathrm{m}}$ and fixed $V_{\max }$ ); 2) fixing both of them (both fixed); 3) allowing full computational liberty for both parameters with optimization of correlation quality (best $R^{2}$ ); 4) using a combined model that involved minimization of the differences between the calculated and assumed parameter values; and 5) using the highest possible correlation coefficient (optimal fit).

Confocal Imaging. MDCK-WT cells were seeded at a density of 25,000 cells per well on a thin bottom 96-well plate (SCREENSTAR; Greiner Bio-One) and incubated for 24 hours. Next, cell cultures were incubated at $37^{\circ} \mathrm{C}$ with $20 \mu \mathrm{M}$ quercetin solution in HBSS/BSA for 40 minutes. Subsequently, cells were washed briefly with cold PBS and treated with $250 \mu \mathrm{g} / \mathrm{ml} \mathrm{APB}$ and $5 \mu \mathrm{M}$ Hoechst 33342 (nuclear stain) in HBSS for 30 minutes in $37^{\circ} \mathrm{C}$. Following one wash with HBSS, cell plasma membranes were stained for 5 minutes with 500 nM mCLING-ATTO 647 dye (Synaptic Systems). Images were obtained using the LSM 780 confocal microscope (Zeiss) with PlanApochromat 63x/1.4 Oil DIC M27 objective. Nuclear fluorescence was imaged with 405-nm excitation and 415-500 $\mathrm{nm}$ emission range, quercetin-APB conjugate fluorescence was imaged with 490-nm excitation and 516-597 $\mathrm{nm}$ emission range, and plasma membrane fluorescence was imaged with $645-\mathrm{nm}$ excitation and $650-775 \mathrm{~nm}$ emission range.

Statistical Analysis and Curve Fitting. For multiple comparisons, ANOVA was used with $P<0.05$ as the significance limit. Linear regression was performed by the standard least-squares method. 
Nonlinear regression (for exponential and hyperbolic curves) was performed iteratively using the least-squares method and Microsoft Excel Solver plugin with appropriate confidence limit calculations (for $P<0.05$ ) (Brown, 2001).

\section{Results}

Fluorescence Calibration Curves. For our experiments, three flavonoids with identical backbones and different hydroxyl group arrangements were selected (Fig. 1A), which were all able to form fluorescent adducts with APB. Calibration curves for these fluorescent products were strongly linear across a wide range of concentrations (Fig. 1B), allowing us to calculate the fluorescence intensity coefficient (regression slope) for each flavonoid and use these values subsequently to calculate the flavonoid concentration in the experimental samples.

Quercetin Undergoes Chemical Transformation in DMEM Medium. Solutions of quercetin at $5 \mu \mathrm{M}$ concentration in DMEM, HBSS, or distilled water (with or without addition of $2.2 \mathrm{mg} / \mathrm{ml}$ BSA) were incubated at $37^{\circ} \mathrm{C}$ for different time periods, diluted 10 -fold with LB, and fluorescence of the APB adduct was measured (Fig. 1C). The fluorescence values (reflecting the concentration of quercetin available for conjugation with APB) remained stable in all of the solutions except for those in DMEM, where a steady decrease in fluorescence over time was seen (indicating
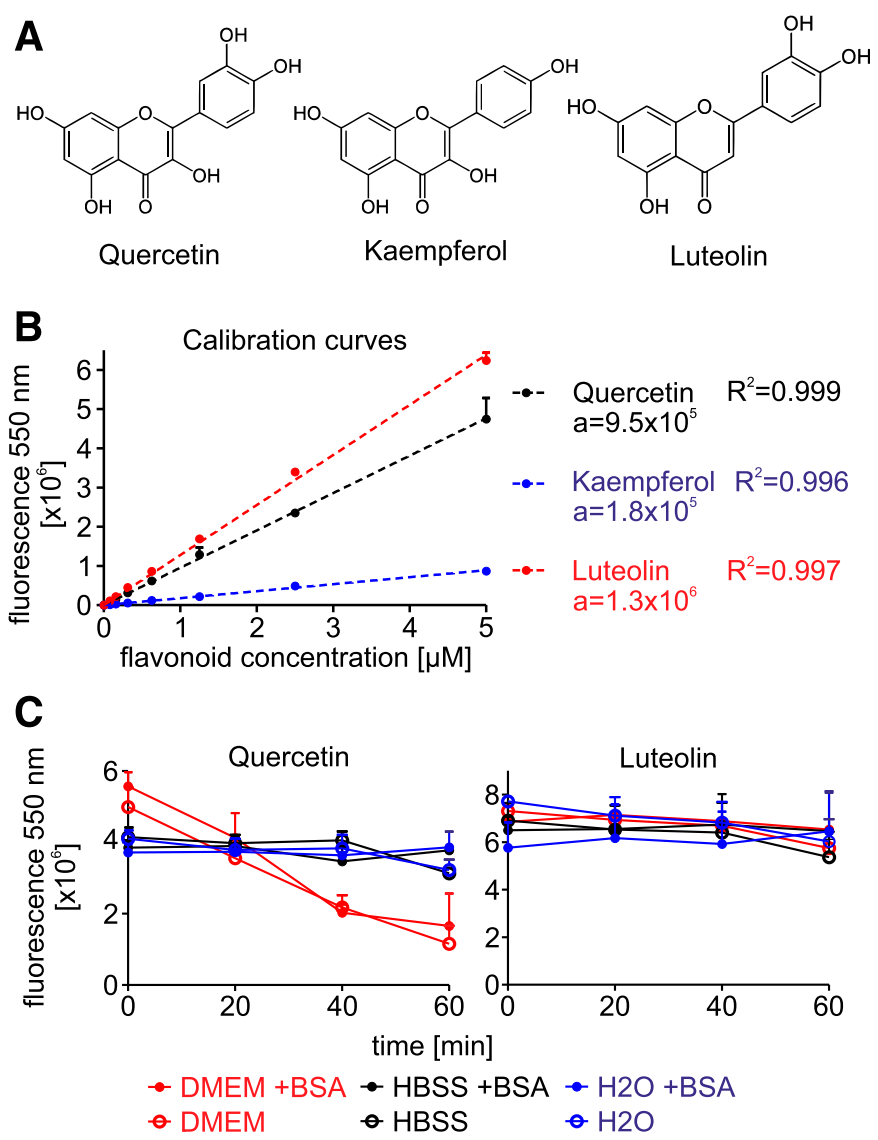

Fig. 1. (A) Structures of the studied flavonoids. (B) Fluorescence calibration curves for the APB conjugates of the studied flavonoids $(n=$ $3 ;$ mean + S.D.) (C) Stability of quercetin and luteolin in various solutions $(n=3 ;$ mean + S.D. $)$ a chemical transformation into a form that cannot react with APB). The presence of BSA did not affect the stability of quercetin. When an analogous experiment was performed for luteolin, the fluorescence values were stable in all of the solutions, indicating no chemical transformation of this flavonoid.

Lysis Buffer Composition Affects Fluorescent Properties of the Flavonoid-APB Conjugate. To optimize the conditions for reliable measurement of intracellular flavonoid concentration, we tested the influence of potential biochemical sources of interference on measured APB conjugate fluorescence. We designed a cell-free experiment where fluorescence was measured for samples in PBS with the same final concentration of $1 \mu \mathrm{M}$ flavonoid and $250 \mu \mathrm{g} / \mathrm{ml}$ APB. These samples differed by the presence or absence of Triton X-100 ( $0.35 \%$ final concentration), BSA $(0.22 \mathrm{mg} / \mathrm{ml})$, and the order of addition of components. The preparation scheme is given in Table 1.

When no Triton X-100 is present (sample APB), conjugate fluorescence for all flavonoids is relatively weak and significantly increases in the presence of BSA (Fig. 2A). On the other hand, the addition of surfactant also increases the fluorescence with regard to the APB sample without BSA; however, this increase is different from the BSA-mediated increase (lower in the case of quercetin and kaempferol, higher in the case of luteolin). Significantly, the impact of Triton X-100 supersedes the impact of BSA: the conjugate fluorescence in the Triton-containing samples is the same regardless of the presence of BSA; therefore, for luteolin, the fluorescence of the Triton X-100 + BSA samples is actually lower than that of the BSA-only samples. This effect is independent of the order of addition of APB, Triton X-100, and BSA; the final fluorescence intensity in the presence of Triton (samples APB/Triton, Triton/APB, and LB both with and without BSA) is always the same.

Since it was apparent from the aforementioned results that BSA and Triton X-100 affect the fluorescent properties of flavonoid-APB conjugates by different and mutually exclusive mechanisms, we performed concentration titration of Triton X-100 (Fig. 2B) and BSA (Fig. 2C) at constant flavonoid and APB concentrations. There is a sigmoidal dependence between Triton concentration and fluorescence, suggesting a connection to its surfactant properties and phase transitions. Indeed, the abrupt rise in fluorescence intensity starts at concentrations close to the critical micelle concentration for Triton X-100 (Rharbi et al., 1999). On the other hand, the dependence between BSA concentration and fluorescence is hyperbolic, suggesting saturational binding with apparent equilibrium at the 1:2 molar ratio of BSA to flavonoid.

Generation and Fate of Intracellular Flavonoid-APB Conjugates. MDCK-WT cells were loaded with quercetin in HBSS/BSA, incubated for 30 minutes at $37^{\circ} \mathrm{C}$ in HBSS/APB, stained with Hoechst 33342 (nuclear stain) and mCLINGATTO 647 (plasma membrane stain); subsequently, cells were imaged in the confocal microscope. The quercetin-APB conjugate localizes predominantly to intracellular structures within the cytoplasm (Fig. 3a). To test the stability of quercetin-APB conjugate distribution, cells were also incubated with quercetin and APB as previously described, Subsequently, HBSS/APB was removed, cells were incubated for 1 hour at $37^{\circ} \mathrm{C}$ in HBSS, nuclei and cell membranes were 
TABLE 1

Order of the addition of reaction components in the experiment on the influence of lysis buffer composition on flavonoid-APB conjugate fluorescence

\begin{tabular}{|c|c|c|}
\hline \multirow{2}{*}{$\begin{array}{l}\text { Sample } \\
\text { Name }\end{array}$} & \multicolumn{2}{|l|}{ Mix and Incubate the Solution for 10 Minutes at Room Temperature } \\
\hline & Solution 1 & Solution 2 \\
\hline APB & $200 \mu \mathrm{l}$ APB $(500 \mu \mathrm{g} / \mathrm{ml})+10 \mu \mathrm{l}$ of $40 \mu \mathrm{M}$ flavonoid solution (in PBS or PBS/BSA) & $200 \mu \mathrm{l}$ PBS \\
\hline $\mathrm{APB} /$ Triton & $200 \mu \mathrm{l}$ APB $(500 \mu \mathrm{g} / \mathrm{ml})+10 \mu \mathrm{l}$ of $40 \mu \mathrm{M}$ flavonoid solution (in PBS or PBS/BSA) & $\begin{array}{l}200 \mu \mathrm{l} \text { Triton X-100 } \\
(0.7 \%)\end{array}$ \\
\hline Triton/APB & $200 \mu \mathrm{l}$ Triton X-100 $(0.7 \%)+10 \mu \mathrm{l}$ of $40 \mu \mathrm{M}$ flavonoid solution (in PBS or PBS/BSA) & $\begin{array}{l}200 \mu \mathrm{l} \mathrm{APB} \\
(500 \mu \mathrm{g} / \mathrm{ml})\end{array}$ \\
\hline $\mathrm{LB}$ & $\begin{array}{l}200 \mu \mathrm{l} \text { APB }(500 \mu \mathrm{g} / \mathrm{ml})+200 \mu \mathrm{l} \text { Triton X-100 }(0.7 \%)+10 \mu \mathrm{l} \text { of } 40 \mu \mathrm{M} \text { flavonoid } \\
\text { solution (in PBS or PBS/BSA) }\end{array}$ & No addition \\
\hline
\end{tabular}

stained as previously described, and cells were imaged. No significant changes in amount and distribution of the fluorescent conjugate were seen (data not shown).

Since the previous result suggests that the flavonoid-APB conjugate cannot permeate the cell membrane (in contrast to its substrates, APB, and flavonoid molecules, which diffuse passively through biologic membranes), we performed a kinetic experiment to test this hypothesis. MDCK-WT cells were preincubated for 40 minutes at $37^{\circ} \mathrm{C}$ with $15 \mu \mathrm{M}$ quercetin in HBSS/BSA. Subsequently, cells were washed with cold PBS and incubated with HBSS or HBSS/APB. In some cases, HBSS/APB was replaced with HBSS after 10 minutes. After specific time periods, supernatant was removed, cells were washed with cold PBS, lysed with LB, and fluorescence was

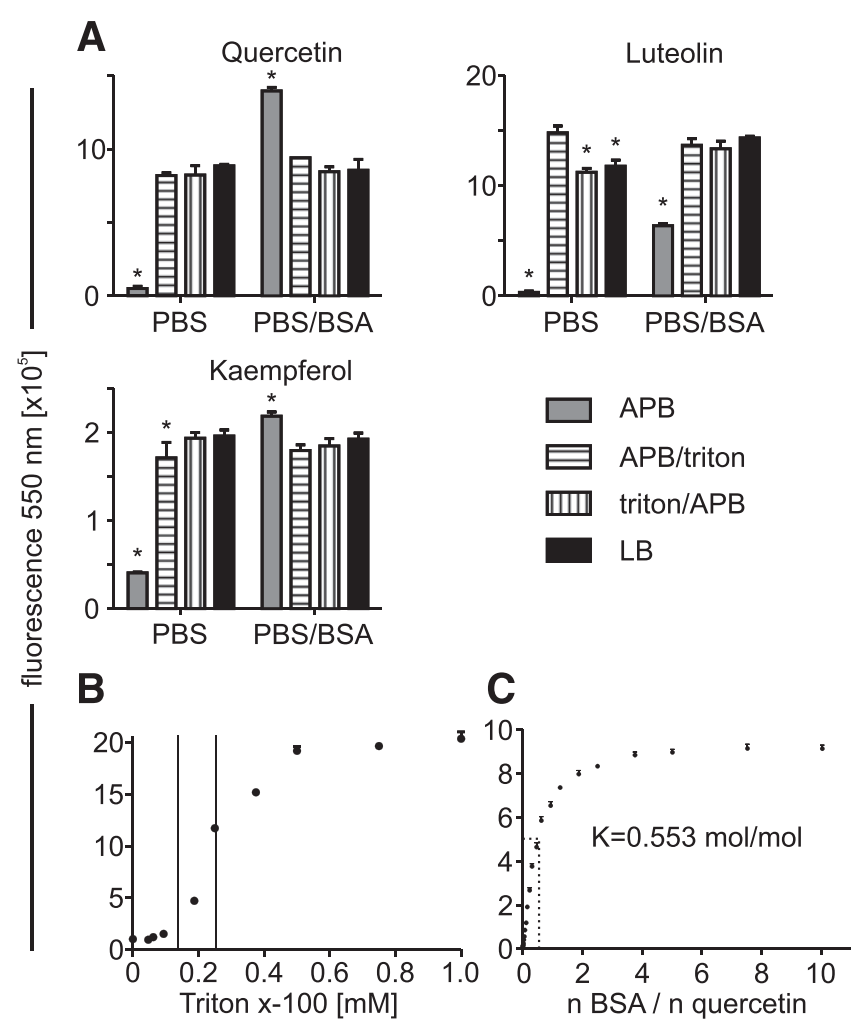

Fig. 2. (A) Fluorescence intensity of the flavonoid-APB conjugates under different reaction conditions $[n=3$; mean + S.D.; $* P<0.05$ (Dunnett's post hoc test vs. LB in PBS/BSA)]. (B) Dependence of the fluorescence intensity of quercetin-APB on the Triton X-100 concentration (vertical lines denote the range of Triton X-100 critical micelle concentration) (Rharbi et al., 1999). (C) Dependence of fluorescence intensity of quercetinAPB on BSA concentration. measured. Concurrently, supernatant was mixed with an equal volume of $2 \mathrm{X}$ concentrated $\mathrm{LB}$ and its fluorescence was also measured. Figure $3 \mathrm{~B}$ shows that the intracellular concentration of quercetin decreases in cells incubated in
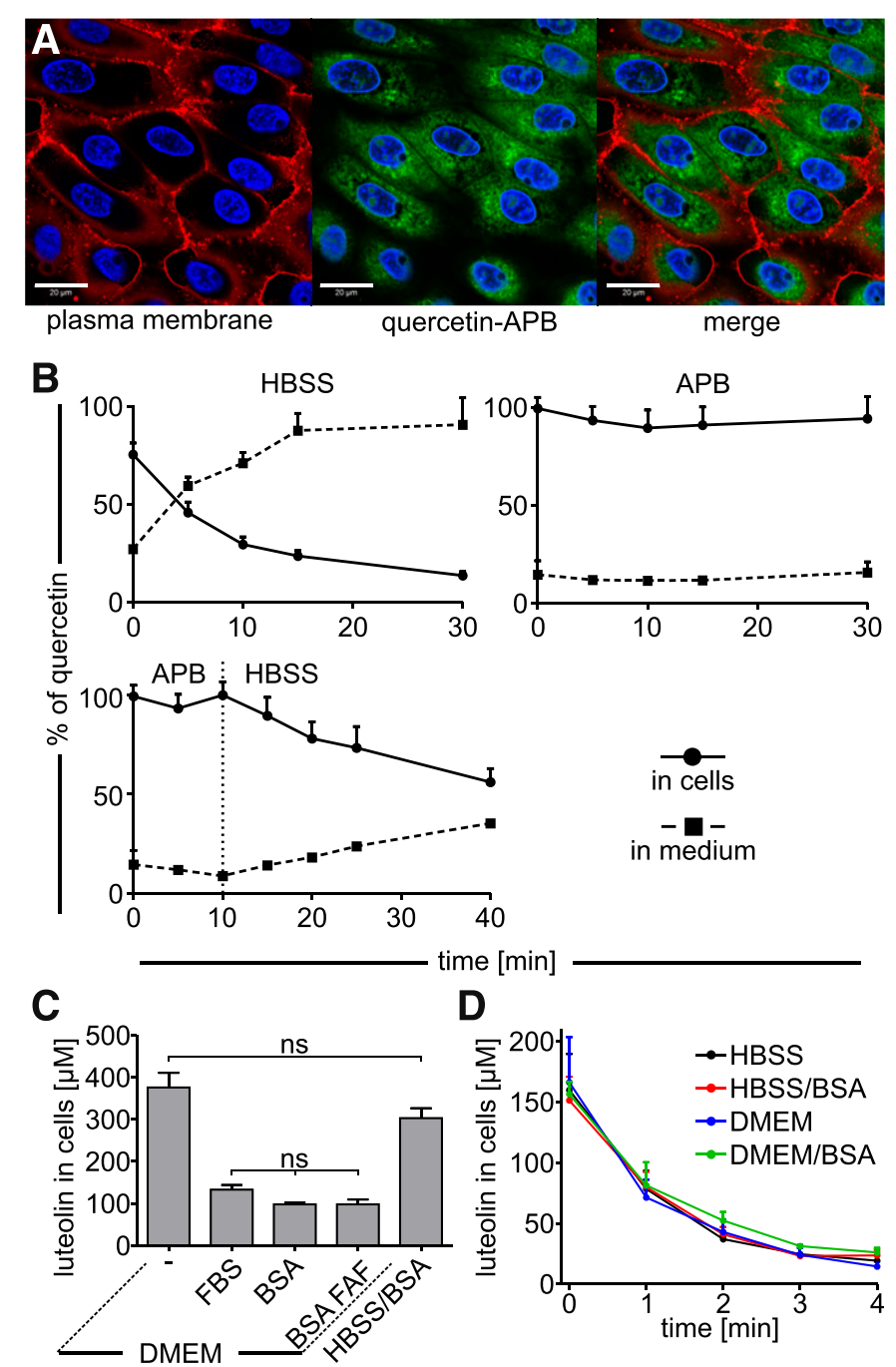

D

Fig. 3. (a) Localization of the quercetin-APB conjugate (green) in MDCKWT cells: plasma membrane stained red (mCLING-ATTO 647); nuclei stained blue (Hoechst 33342); scale bar, $20 \mu \mathrm{m}$. (B) Intra-/extracellular concentration of APB-quercetin during efflux in the presence and absence of extracellular APB $(n=6$; mean + S.D. $)$ (C) Equilibrium concentration of luteolin inside MDCK-WT cells in the presence of $20 \mu \mathrm{M}$ luteolin and other additives outside the cell $[n=6$; mean + S.D.; non-significant $P>$ 0.05 (Tukey's post hoc test)]. (D) Passive efflux of luteolin to different media $(n=6$; mean + S.D. $)$ 
HBSS (and the extracellular concentration increases concurrently); reflecting passive efflux from the preloaded cells. On the other hand, when APB is present it prevents this efflux and stabilizes the intracellular quercetin concentration, attesting to the fast kinetics of the APB-flavonoid reaction and membrane-impermeable character of the resulting conjugate. When APB is removed after 10-minute incubation, quercetin efflux resumes, albeit at a much reduced ratio, reflecting a reduced amount of unconjugated quercetin in the cell.
A

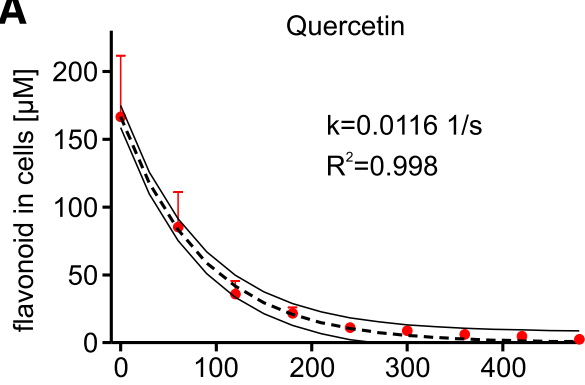

B

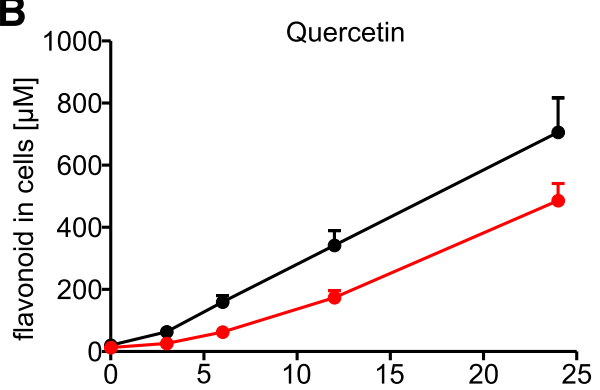

C

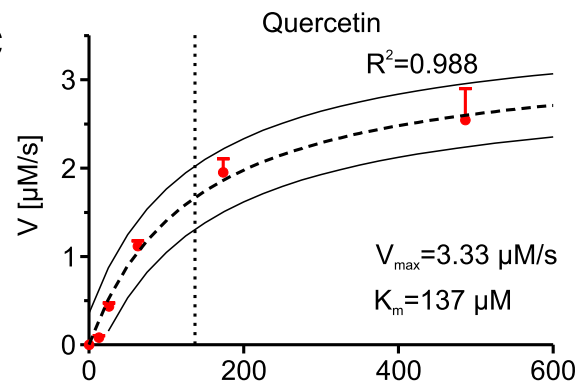

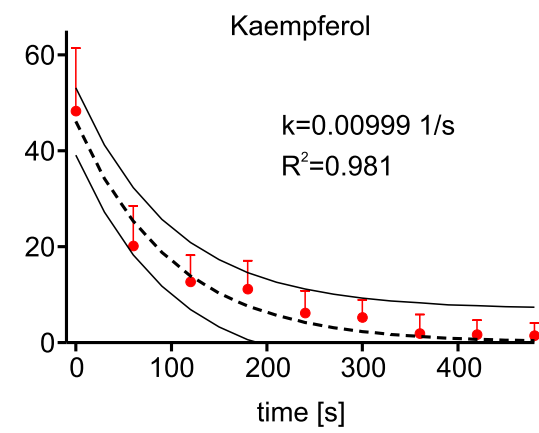
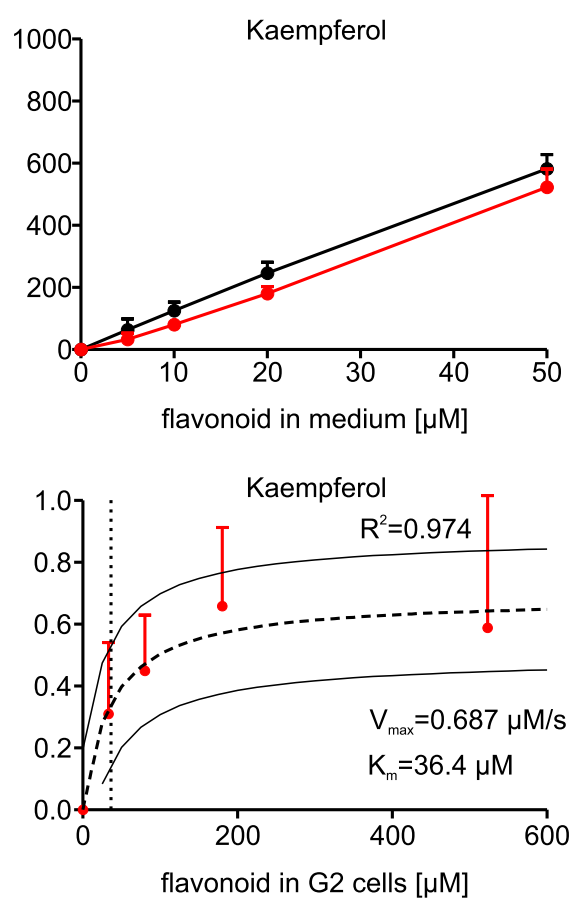
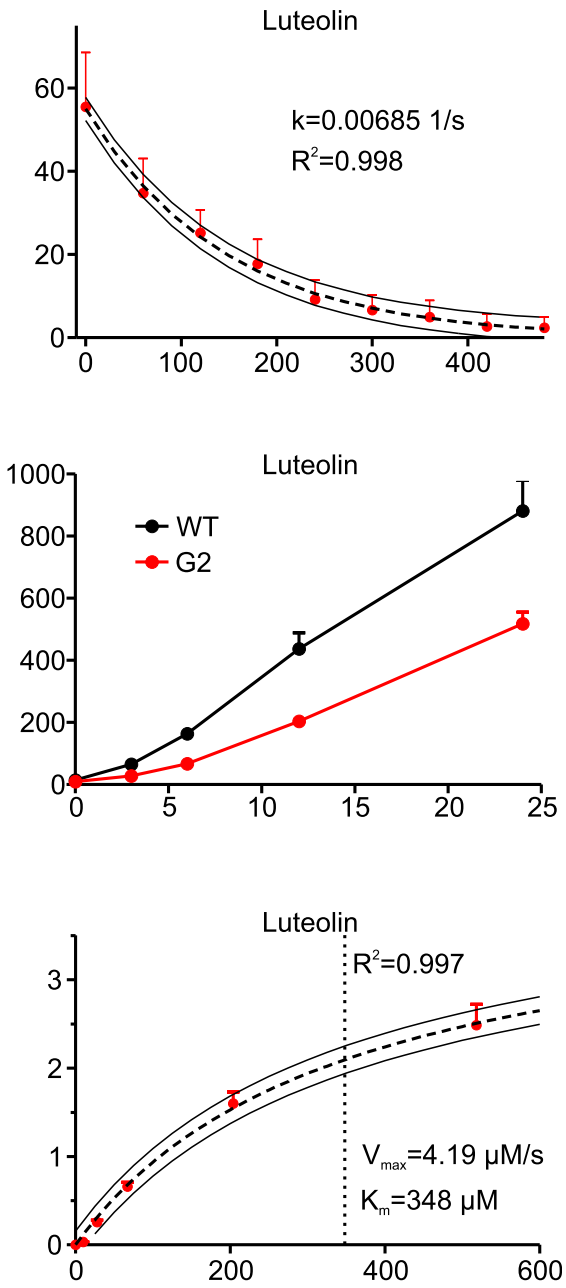
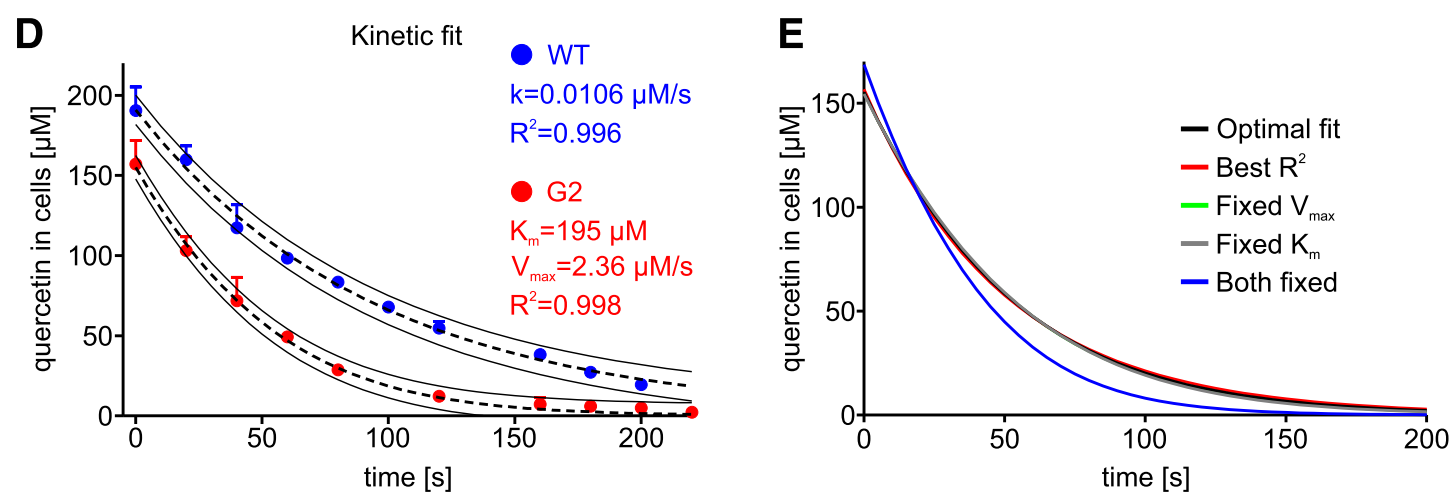

Fig. 4. (A) Passive efflux of flavonoids from MDCK-WT ( $n=6-11$; mean + S.D.; dashed lines denote fitted first-order kinetic curve; solid lines denote $95 \%$ confidence intervals). (B) Equilibrium intracellular concentrations of flavonoids in MDCK-WT and MDCK-G2 cells ( $n=16,3$, and 8, respectively; mean + S.D.) (C) Michaelis-Menten plots for tested flavonoids $(n=16,3$, and 8, respectively; mean + S.E.M.; dashed lines denote fitted MichaelisMenten curve; solid lines denote $95 \%$ confidence intervals; vertical dotted lines denote $K_{\mathrm{m}}$ ). (D) Efflux of quercetin from MDCK-WT and MDCK-G2 cells $[n=3$; mean + S.D.; dashed lines denote fitted first-order kinetic curve (for WT cells) or combined model optimal fit curve (for G2 cells); solid lines denote 95\% confidence intervals]. (E) Fitted curves for different statistical models (described in Materials and Methods). 
Medium Composition Affects Equilibrium Concentration of Flavonoids in a Cell. While we proved that the presence of albumin affects the fluorescence of the flavonoidAPB conjugate, it was unclear whether its presence in a cellular transport model can also impact passive flavonoid diffusion and equilibrium distribution. Therefore, we tested the impact of extracellular medium composition on equilibrium concentration of luteolin (selected as the model flavonoid in this experiment, since the previously shown propensity of quercetin to chemical transformation in DMEM would add a confounding variable to the analysis). We demonstrate that the presence of extracellular protein decreases the intracellular flavonoid concentration at equilibrium (Fig. 3C), with the effect being dependent mainly on albumin concentration and independent of the presence of nonprotein components: the concentration is identical if standard (lipid-containing) or fatty acid-free BSA is used, and in the case of FBS (where BSA accounts for $60 \%$ of all protein by weight) it is only slightly increased. Surprisingly, the presence of other medium components also has a drastic impact on equilibrium flavonoid concentration: it is much higher in HBSS than in DMEM. At the same time, neither protein/BSA nor other medium components influence the rate of passive diffusion itself (Fig. 3D): when equilibrium is reached in HBSS/BSA and the medium is subsequently exchanged, the rate of decrease of intracellular flavonoid concentration is the same. Therefore, further equilibrium experiments were performed in HBSS/BSA.

Measurement of Kinetic Transport Parameters for Flavonoids. Intracellular concentration of flavonoids in MDCK-WT cells was monitored over time during efflux from preloaded cells. Exponential first-order decay curves were fitted to experimental points to calculate the passive efflux constant $k$ (Fig. 4A). The values obtained from nonlinear regression were further used in subsequent calculations of enzyme-mediated transport parameters.

The $K_{\mathrm{m}}$ and $V_{\max }$ values of ABCG2-mediated active transport of the investigated flavonoids were derived from experiments involving comparison of equilibrium intracellular flavonoid concentration in MDCK-WT and MDCK-G2 cells (Fig. 4B). Michaelis-Menten curves were fitted to experimental points (intracellular concentration in MDCK-G2 cells vs. ABCG2-mediated transport velocity) and the $K_{\mathrm{m}}$ and $V_{\max }$ values were calculated by nonlinear regression for each flavonoid (Fig. 4C).

Alternative Derivation of Active Efflux Parameters from Kinetic Data. Since the kinetics of flavonoid efflux from ABCG2-expressing cells is dependent on ABCG2 efficiency and substrate affinity, the shape of the efflux curve (intracellular substrate concentrations at various times after cell preloading and during subsequent efflux to substrate-free extracellular solution) can also be used to derive $K_{\mathrm{m}}$ and $V_{\max }$ parameters for ABCG2. However, these parameters do not have a strong impact on the overall shape of the curve and their calculation from iterative nonlinear regression is inherently nonexact. These approximations are made even more difficult by the interconnected character of the relevant parameters; therefore, this method is more appropriate as confirmation of the applicability of otherwise known parameter values (e.g., calculated from equilibrium experiments, as in our case) to efflux conditions. We demonstrate this with the experimental data on quercetin transport (efflux curves from
MDCK-WT and MDCK-G2 cells at identical extracellular quercetin concentrations during equilibrium), which are used to calculate the $K_{\mathrm{m}}$ and $V_{\max }$ values by partially restricted curve fitting. Figure 4D shows the results (regression curve and its confidence intervals) for nonlinear regression by the optimal fit, which are in relatively good accordance with the equilibrium-derived values for this substrate. Table 2 and Fig. 4E show a comparison of all of the regression approaches, with the least-restricted approach (best $R^{2}$ ) being the only one to yield highly divergent values; further underlining the need for knowledge-derived mathematical boundary conditions in this method and proving the value of efflux-type experiments as confirmatory rather than exploratory.

Enzyme Kinetics of ABCG2-Mediated Transport Inhibition. To further validate our method of kinetic parameter derivation from equilibrium experiments for practical applications, we decided to test the influence of known ABCG2 transport inhibitors (kaempferol and 5D3 antibody) on the calculated $V_{\max }$ and $K_{\mathrm{m}}$ values for quercetin. In these experiments, MDCK-WT and MDCK-G2 cells were preincubated for 15 minutes with different inhibitor concentrations in HBSS/ BSA and subsequently incubated with quercetin at various concentrations in HBSS/BSA (in the continuing presence of inhibitors) for 40 minutes. Further measurements and calculations were analogous to those described previously for the equilibrium experiments. Figure 5A shows the measured intracellular concentrations used to calculate the difference between intracellular quercetin concentrations in both cell lines. Figure 5B shows the derived Michaelis-Menten curves. Kaempferol is a typical competitive inhibitor; therefore, it should increase the $K_{\mathrm{m}}$ value without changing the $V_{\max }$ value, which is supported by the shape of the experimental curves (the calculated apparent $K_{\mathrm{m}}$ values for 3 and $10 \mu \mathrm{M}$ kaempferol inhibitions are 586 and $1094 \mu \mathrm{M}$, respectively). On the other hand, 5D3 antibody (which binds to an extracellular epitope, blocking the ABCG2 protein in one conformation in its activity cycle) is a typical noncompetitive inhibitor, and again the shape of the experimental curves bears out this assumption (the calculated apparent $V_{\max }$ values for 1 and $5 \mu \mathrm{g} / \mathrm{ml}$ 5D3 inhibitions are 1.07 and $0.422 \mu \mathrm{M} / \mathrm{s}$, respectively).

\section{Discussion}

Flavonoids are abundant dietary components with important bioactivities in the human body. Their targets and modes of action are being extensively characterized and investigated, and practical therapeutic applications (both medicinal and nutriceutical) are being developed; therefore, questions about their pharmacokinetic characteristics are highly topical (AhnJarvis et al., 2019; Maher, 2019; Ye et al., 2019). It is well

\section{TABLE 2}

Kinetic parameters for ABCG2-mediated transport of quercetin calculated from the direct efflux experiment using different curve fitting options. Asterisk denotes values fixed in this calculation, derived previously from the equilibrium experiment.

\begin{tabular}{lrll}
\hline Fitting Option & $K_{\mathrm{m}}$ & $V_{\max }$ & $R^{2}$ \\
\hline & $\mu M$ & $\mu M / s$ & \\
Fixed $V_{\max }$ & 311 & $3.33^{*}$ & 0.9982 \\
Fixed $K_{\mathrm{m}}$ & $137^{*}$ & 1.86 & 0.9973 \\
Best $R^{2}$ & 223,417 & 1871 & 0.9987 \\
Optimal fit & 195 & 2.36 & 0.9978 \\
Both fixed & $137^{*}$ & $3.33^{*}$ & 0.9646 \\
\hline
\end{tabular}



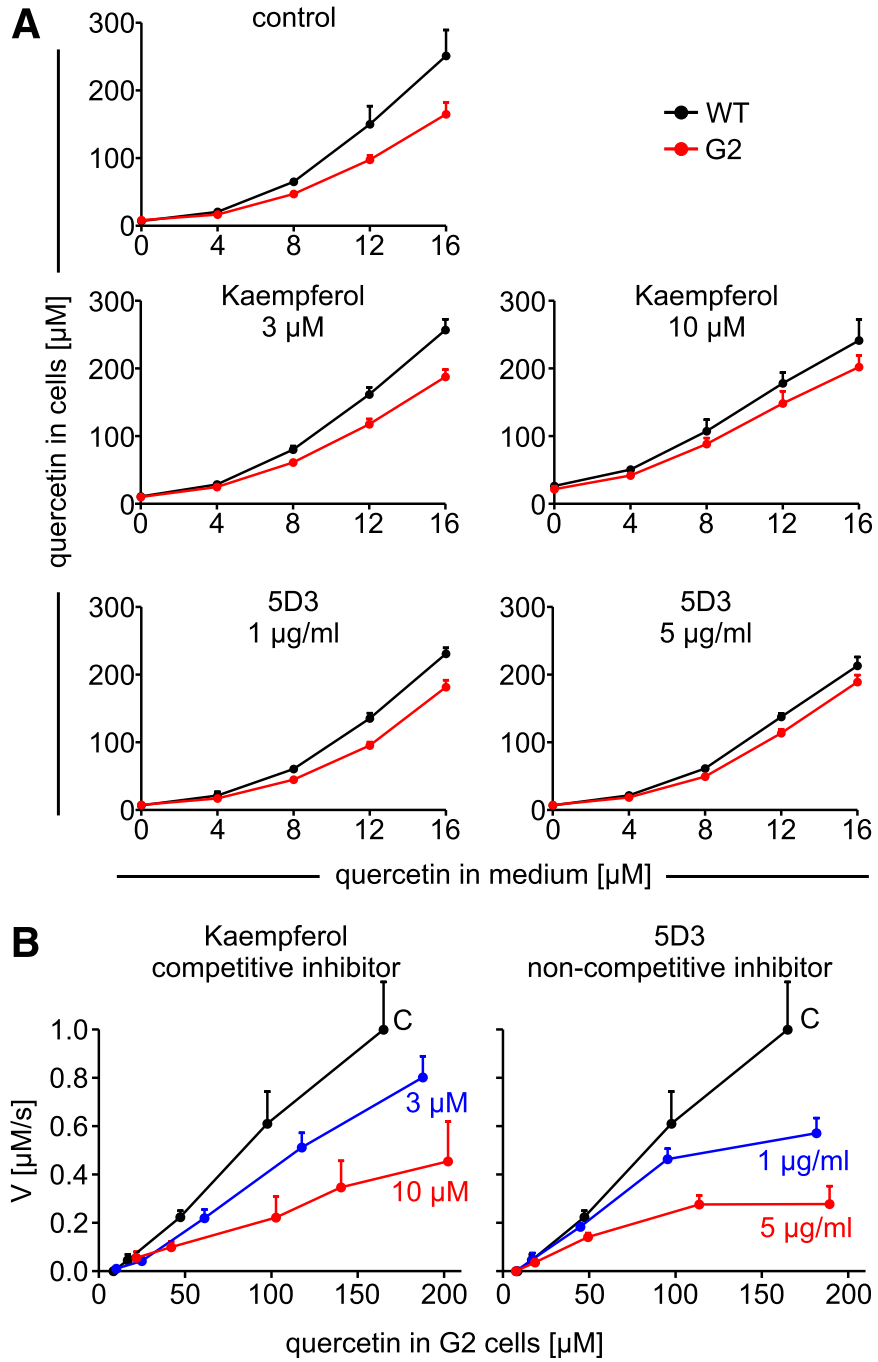

Fig. 5. (A) Equilibrium intracellular concentrations of quercetin in MDCK-WT and MDCK-G2 cells in the absence and presence of inhibitors $(n=6$; mean + S.D.). (B) Michaelis-Menten plots for quercetin transport in the presence of inhibitors ( $\mathrm{C}$ denotes control; $n=6$; mean + S.E.M.).

established that $\mathrm{ABC}$ transporter activity in barrier tissues plays a crucial role in the bioavailability and biodistribution of flavonoids and that ABCG2 is a key player in this respect (Yang et al., 2012; Gonzales et al., 2015; Peña-Solórzano et al., 2017). Thus, reliable and broadly available methods for characterizing the interactions between flavonoids and ABCG2 are of high scientific and practical interest.

2-Aminoethyl diphenylborinate is a reagent that has seen practical use in biomedical laboratories in two entirely divergent applications. On one hand, plant biochemists have used it for colorimetric or fluorimetric detection of flavonoids, mainly in chromatography or microscopy (Saslowsky and Winkel-Shirley, 2001; Filippi et al., 2015). On the other hand, it has been serendipitously identified and used as a specific inhibitor of some mammalian calcium channels (Kovacs et al., 2012). As a fluorogenic substrate, it is especially valuable since it diffuses quickly through biologic membranes and reacts very quickly with its target compounds. Matteini et al. (2011) characterized the probable mechanism of reaction between APB and katechol or ketoenol groups in flavonoid molecules. Significantly, this has led us to the observation that no major mammalian metabolites seem to possess the required hydroxyl group/aromatic ring arrangement; therefore, the background fluorescence in mammalian cells due to conjugation of endogenous compounds with APB is negligible. Based on this, in 2014 we proposed and patented the concept of using APB to measure intracellular concentration of exogenously added flavonoid in mammalian cells and applying this to kinetic studies of $\mathrm{ABC}$ transporters (Studzian and Pulaski, 2017). The present paper elaborates on this concept and presents practical results obtained using this technique. Recently, there have been other reports about the application of APB for measuring exogenous flavonoids in animal cells, e.g., flow cytometric assays in cell lines (Grootaert et al., 2016) as well as in vivo assays in unicellular organisms (Ferrara and Thompson, 2019).

To reliably characterize the transport parameters of a transporter protein in whole cells, the right cellular model is crucial. Therefore, for our study we selected MDCKII, a polarized epithelial cell line widely used as a model in transporter research (Mukkavilli et al., 2017; Szilagyi et al., 2019). While sublines of these cells overexpressing human ABCG2 have been available for some time, we took advantage of the recent commercial availability of a unique variant (huMDCKII) in which the endogenous canine multidrug transporters (cfABCB1 and cfABCG2) were knocked out by genetic engineering, thus removing an important confounding factor in quantitative analysis. This model retains the mammalian cellular environment (which has been demonstrated to be important for multidrug transporter activity) (Telbisz et al., 2007; Neumann et al., 2017) preferable to heterologous expression in lower eukaryotes (where the unpredictable background from endogenous transporters also precludes quantitative studies on nonstandard substrates).

The goal of our study was the derivation of kinetic parameter values for ABCG2-mediated flavonoid transport; therefore, a large part of our work was devoted to controls, validations, and verifications of the experimental setup. APB is unique in that it passes membranes easily; however, the conjugate with a flavonoid molecule is charged and does not (Matteini et al., 2011). We demonstrate here that this conjugate is also not a substrate for any active or passive export systems; once formed in the cell, it is well retained inside. This feature, together with the relatively quick conjugation kinetics, results in the ability to freeze frame the intracellular concentration of a flavonoid for convenient kinetic measurements with good time resolution. Moreover, we were able to show that the conjugates are stable and have a broad linear fluorescence range, both preconditions for quantitative measurements. Since standard assay methods for polyphenol transport in mammalian systems involve their isolation and/or separation from the biologic matrix (usually, chromatography linked to mass spectrometric detection) (Li et al., 2003; Hu et al., 2012), our technique is both much less labor and time intensive and less prone to technical artifacts.

Within careful validation of experimental parameters, we needed to investigate the effect of medium and lysis buffer components on flavonoid distribution as well as conjugate formation and fluorescence. Flavonoids are known to bind to certain proteins, including serum albumin (Dufour and Dangles, 2005; Yang et al., 2008). Therefore, our results showing that inclusion of BSA in extracellular medium leads to a shift 
in the equilibrium distribution of flavonoid molecules inside and outside of cells were expected. It is known that flavonoid binding changes the fluorescent properties (increases the quantum yield) (Sengupta and Sengupta, 2003). We show that the APB-flavonoid conjugate also binds to albumin, resulting in enhanced fluorescence output, which allows us to characterize the binding equilibrium. On the other hand, measurement of intracellular flavonoid concentration involves cell lysis, and thus is necessarily done in the presence of detergents; we found that Triton X-100 also increases fluorescence emission of the conjugate, especially at concentrations above its critical micelle concentration. These results support the hypothesis of quantum yield increase by spatial stabilization (increased ring rigidity) and protection of the fluorophore moiety in the conjugate molecule, as was suggested for endogenous quercetin fluorescence (Sengupta and Sengupta, 2003). This is further supported by the fact that if albumin and Triton X-100 are present concurrently, fluorescence output is determined exclusively by the detergent, attesting to its ability to extract the conjugate molecule from albumin and incorporate it into the micelle.

Interestingly, quercetin showed a marked time-dependent loss of the ability to form the fluorescent conjugate with APB when incubated in standard cell-culture medium (DMEM). It has been previously reported that sodium bicarbonate in cell culture medium negatively impacts quercetin stability ( $\mathrm{Hu}$ et al., 2012). We show that this effect is limited to quercetin among studied flavonoids, independent of the presence of albumin, and easily remedied in transport studies by using buffered saline in the extracellular environment.

In a complex cellular system, active membrane transport kinetics is determined by many facets of substrate distribution. We show that the intracellularly formed conjugate is retained in the cytoplasm (as visualized microscopically) and can be reliably and easily used to calculate the (average) absolute intracellular concentration of the flavonoid at the time point of conjugation, serving as a proxy for free substrate concentration in calculations of passive diffusion and transporter enzyme kinetics. As a necessary starting point, we used this approach to calculate first-order passive diffusion constants by curve fitting of decreasing intracellular flavonoid concentration in preloaded cells (a standard biochemical approach when a reliable quantitative assay is available for intracellular substrates) (e.g., Ortega and Rodríguez-Navarro, 1985). Interestingly, luteolin had the lowest passive diffusion rate of the studied molecules, despite theoretically not being the most hydrophilic molecule. In vectorial transport assays through a Caco-2 monolayer, luteolin has previously been shown to have higher passive permeability than quercetin or keampferol (Fang et al., 2017), undescoring the differences inherent in various cellular models using in vivo assays.

The main advantage of the method presented in this paper is the combination of easy and reliable determination of intracellular substrate concentrations with mathematical calculation of enzyme constants based on an additive transport model (concurrent passive and active effluxes). Thus, using the equilibrium approach we were able to derive absolute values of both the apparent $K_{\mathrm{m}}$ (in units of average intracellular substrate concentration) and apparent $V_{\max }$ (in units of reaction velocity per whole cell, independent of total protein concentration). These values are useful both for comparisons of affinity and transport efficiency between various flavonoids as well as for phenomenological flux calculations for an individual cell (e.g., in pharmacokinetic modeling of barriers composed of cell monolayers). Due to difficulties in finding a correct cellular model and technical problems with assays, there is a very limited body of literature on directly derived kinetic constants of ABCG2-mediated flavonoid transport. An et al. (2011) performed vectorial transport experiments through MDCKII monolayers (with heterologous overexpression of murine Abcg2) and concluded that kaempferol is a high-affinity, low-capacity transport substrate. This agrees with our results, even though the apparent $K_{\mathrm{m}}$ value calculated in the present study $(\sim 36 \mu \mathrm{M})$ was more than an order of magnitude higher than that calculated by An et al. (2011) $(\sim 1.3 \mu \mathrm{M})$; this may be due both to differences in experimental design and species difference between mouse and human transporters. We also confirm the designation of luteolin as a relatively low-affinity, high-capacity ABCG2 substrate (Sadowska-Bartosz et al., 2016).

The capacity of the APB method to precisely assay intracellular flavonoid concentration at defined time points (thanks to the near-instantaneous reaction kinetics) allowed us to test an alternative practical approach to the derivation of kinetic constants. Instead of measuring equilibrium concentrations and describing them in a zero-net-flux model, we can also perform a direct kinetic assay of substrate export to extracellular space, where its concentration is negligible (due to the high dilution coefficient). Of course, direct measurements at precise time points are cumbersome in practice (because transport is relatively fast, short time periods after initiation are required) and require a complex mathematical approach due to the need to concurrently model two fluxes in the same direction (active and passive); however, on the other hand, direct detection of substrate concentration changes is a reliable, artifact-proof approach to transport kinetics. By a restricted regression method (optimal fit), we were able to obtain similar values of kinetic parameters as with the equilibrium approach, lending credence to this procedure. This method is conceptually analogous to the standard insideout vesicle assay, which depends on monitoring and mathematically fitting the changes of substrate concentration inside the vesicle (analog of extracellular space) during unidirectional transport. Thus, we are using similar mathematical flux modeling tools to derive enzyme kinetic constants as those that have been used in all major descriptions of ABCG2 activity, e.g., to derive the $K_{\mathrm{m}}$ and $V_{\text {max }}$ values for its major endogenous substrate urate (Nakayama et al., 2011). However, the advantage of our model is the physiologic cellular setting (intact whole cell and no need to account for leakage from unsealed vesicles).

We were able to validate another application of our method, which provides a significant advantage to current state-of-theart methods. We tested the inhibitory potency and kinetic derivation of the biochemical mechanism of inhibition for two known ABCG2 blockers: kaempferol as a competitive inhibitor (An et al., 2011) and 5D3 antibody as a noncompetitive inhibitor (Telbisz et al., 2012; Studzian et al., 2015). Our curve-fitting procedure yielded expected results, confirming the validity of this approach in classifying inhibitors. At the same time, this method has obvious advantages for practical pharmacokinetic purposes, since inhibition parameters derived in whole cells-in the presence of all intracellular components-are going to be much more physiologically 
relevant in modeling drug distribution in tissues and whole organisms, especially with regard to barrier tissues where ABCG2 function is crucial. Since the equilibrium assay that we describe is easily amenable to miniaturization and automation, this is also a suitable approach for inhibitor screening that yields more relevant kinetic information than just inhibitory potency at individual concentrations, which is an avenue of further studies that we intend to pursue.

In conclusion, we developed and validated a biochemical assay for enzyme kinetics of ABCG2-mediated transport of natural flavonoids. It is interesting, both for mechanistic studies of substrate recognition by this still insufficiently understood $\mathrm{ABC}$ transporter and practically for studies of the pharmacokinetic relationship between ABCG2 (especially in its role as a barrier tissue gatekeeper) and dietary flavonoids. Polyphenol compounds are natural (plant-derived) food products that also see increasing use as nutriceuticals, which are recommended for a large variety of ailments and age-related disorders due to their antioxidant capacity. Precise knowledge regarding their interactions with their main active transporter provides practical indications not just about their absorption, distribution, metabolism, and excretion parameters, but also about potential food-drug interactions and personalized applications. The easy automation of this method and its relatively low cost make it ideal for substrate screening and structure-activity relationship studies (even quantitative structure-activity relationships since the calculations result in exact kinetic parameter values), which is our current further research objective.

\section{Acknowledgments}

We thank J. Dastych for access to crucial equipment and G. Bartosz for valuable discussions.

\section{Authorship Contributions}

Participated in research design: Rozanski, Studzian, Pulaski.

Conducted experiments: Rozanski, Studzian.

Performed data analysis: Rozanski, Studzian, Pulaski.

Wrote or contributed to the writing of the manuscript: Rozanski, Studzian, Pulaski.

\section{References}

Ahn-Jarvis JH, Parihar A, and Doseff AI (2019) Dietary flavonoids for immunoregulation and cancer: food design for targeting disease. Antioxidants 8:202.

An G, Gallegos J, and Morris ME (2011) The bioflavonoid kaempferol is an Abcg2 substrate and inhibits Abcg2-mediated quercetin efflux. Drug Metab Dispos 39: 426-432.

Brown AM (2001) A step-by-step guide to non-linear regression analysis of experimental data using a Microsoft Excel spreadsheet. Comput Methods Programs Biomed 65:191-200.

Dufour C and Dangles O (2005) Flavonoid-serum albumin complexation: determination of binding constants and binding sites by fluorescence spectroscopy. Biochim Biophys Acta 1721:164-173.

Ernst O and Zor T (2010) Linearization of the Bradford protein assay. $J$ Vis Exp 38 1918 Available from: 10.3791/1918.

Fang Y, Cao W, Xia M, Pan S, and Xu X (2017) Study of structure and permeability relationship of flavonoids in Caco-2 cells. Nutrients 9:1301.

Ferrara BT and Thompson EP (2019) A method for visualizing fluorescence of flavonoid therapeutics in vivo in the model eukaryote Dictyostelium discoideum. Biotechniques 66:65-71.

Filippi A, Petrussa E, Peresson C, Bertolini A, Vianello A, and Braidot E (2015) In vivo assay to monitor flavonoid uptake across plant cell membranes. FEBS Open Bio 5:748-752

Ford RC and Beis K (2019) Learning the ABCs one at a time: structure and mechanism of ABC transporters. Biochem Soc Trans 47:23-36.

Fraga CG, Croft KD, Kennedy DO, and Tomás-Barberán FA (2019) The effects of polyphenols and other bioactives on human health. Food Funct 10:514-528.

Gantner ME, Peroni RN, Morales JF, Villalba ML, Ruiz ME, and Talevi A (2017) Development and validation of a computational model ensemble for the early detection of BCRP/ABCG2 substrates during the drug design stage. $J$ Chem Inf Model 57:1868-1880.
Ge S, Yin T, Xu B, Gao S, and Hu M (2016) Curcumin affects phase II disposition of resveratrol through inhibiting efflux transporters MRP2 and BCRP. Pharm Res 33: $590-602$

Giacomini KM, Huang SM, Tweedie DJ, Benet LZ, Brouwer KL, Chu X, Dahlin A, Evers R, Fischer V, Hillgren KM, et al.; International Transporter Consortium (2010) Membrane transporters in drug development. Nat Rev Drug Discov 9: $215-236$

Glavinas H, Kis E, Pál A, Kovács R, Jani M, Vági E, Molnár E, Bánsághi S, Kele Z, Janáky T, et al. (2007) ABCG2 (breast cancer resistance protein/mitoxantrone resistance-associated protein) ATPase assay: a useful tool to detect drugtransporter interactions. Drug Metab Dispos 35:1533-1542.

Gonzales GB, Smagghe G, Grootaert C, Zotti M, Raes K, and Van Camp J (2015) Flavonoid interactions during digestion, absorption, distribution and metabolism: a sequential structure-activity/property relationship-based approach in the study of bioavailability and bioactivity. Drug Metab Rev 47:175-190.

Grootaert C, Gonzales GB, Vissenaekens H, Van de Wiele T, Raes K, Smagghe G, and Van Camp J (2016) Flow cytometric method for the detection of flavonoids in cell lines. J Biomol Screen 21:858-865.

$\mathrm{Hu} \mathrm{J}, \mathrm{Xu}$ T, and Cheng Y (2012) NMR insights into dendrimer-based host-guest systems. Chem Rev 112:3856-3891.

Iorio AL, Ros M, Fantappiè O, Lucchesi M, Facchini L, Stival A, Becciani S, Guidi M, Favre C, Martino M, et al. (2016) Blood-brain barrier and breast cancer resistance protein: a limit to the therapy of CNS tumors and neurodegenerative diseases. Anticancer Agents Med Chem 16:810-815

Iriti M, Kubina R, Cochis A, Sorrentino R, Varoni EM, Kabała-Dzik A, Azzimonti B, Dziedzic A, Rimondini L, and Wojtyczka RD (2017) Rutin, a quercetin glycoside, restores chemosensitivity in human breast cancer cells. Phytother Res 31 1529-1538.

Kovacs G, Montalbetti N, Simonin A, Danko T, Balazs B, Zsembery A, and Hediger MA (2012) Inhibition of the human epithelial calcium channel TRPV6 by 2 aminoethoxydiphenyl borate (2-APB). Cell Calcium 52:468-480.

$\mathrm{Li} \mathrm{F}$, Howard KD, and Myers MJ (2017) Influence of P-glycoprotein on the disposition of fexofenadine and its enantiomers. J Pharm Pharmacol 69:274-284.

Li Y, Shin YG, Yu C, Kosmeder JW, Hirschelman WH, Pezzuto JM, and van Breemen RB (2003) Increasing the throughput and productivity of Caco-2 cell permeability assays using liquid chromatography-mass spectrometry: application to resveratrol absorption and metabolism. Comb Chem High Throughput Screen 6:757-767.

Maher P (2019) The potential of flavonoids for the treatment of neurodegenerative diseases. Int J Mol Sci 20:3056.

Mao Q (2008) BCRP/ABCG2 in the placenta: expression, function and regulation. Pharm Res 25:1244-1255.

Matteini P, Agati G, Pinelli P, and Goti A (2011) Modes of complexation of rutin with the flavonoid reagent diphenylborinic acid 2-aminoethyl ester. Monatsh Chem 142 : $885-893$

Mukkavilli R, Jadhav G, and Vangala S (2017) Evaluation of drug transport in MDCKII-wild type, MDCKII-MDR1, MDCKII-BCRP and Caco-2 cell lines. Curr Pharm Biotechnol 18:1151-1158.

Nakayama A, Matsuo H, Takada T, Ichida K, Nakamura T, Ikebuchi Y, Ito K, Hosoya T, Kanai Y, Suzuki H, et al. (2011) ABCG2 is a high-capacity urate transporter and its genetic impairment increases serum uric acid levels in humans. Nucleosides Nucleotides Nucleic Acids 30:1091-1097.

Neumann J, Rose-Sperling D, and Hellmich UA (2017) Diverse relations between ABC transporters and lipids: an overview. Biochim Biophys Acta Biomembr 1859: $605-618$

Ortega MD and Rodríguez-Navarro A (1985) Potassium and rubidium effluxes in Saccharomyces cerevisiae. $Z$ Naturforsch $C$ 40:721-725.

Passamonti S, Terdoslavich R, Franca R, Vanzo A, Tramer F, Braidot E, Petrussa E, and Vianello A (2009) Bioavailability of flavonoids: a review of their membrane transport and the function of bilitranslocase in animal and plant organisms. Curr Drug Metab 10:369-394.

Pedersen JM, Khan EK, Bergström CAS, Palm J, Hoogstraate J, and Artursson P (2017) Substrate and method dependent inhibition of three ABC-transporters (MDR1, BCRP, and MRP2). Eur J Pharm Sci 103:70-76.

Peña-Solórzano D, Stark SA, König B, Sierra CA, and Ochoa-Puentes C (2017) ABCG2/BCRP: specific and nonspecific modulators. Med Res Rev 37:987-1050.

Rharbi Y, Kitaev V, Winnik MA, and Hahn KG (1999) Characterizing aqueous micellar Triton X-100 solutions of a fluorescent model triglyceride. Langmuir 15: $2259-2266$.

Robey RW, Ierano C, Zhan Z, and Bates SE (2011) The challenge of exploiting ABCG2 in the clinic. Curr Pharm Biotechnol 12:595-608.

Sadowska-Bartosz I, Grębowski J, Kepka E, Studzian M, Bartosz G, and Pułaski $~ E$ (2016) ABCB1-overexpressing MDCK-II cells are hypersensitive to 3-bromopyruvic acid. Life Sci 162:138-144.

Saslowsky D and Winkel-Shirley B (2001) Localization of flavonoid enzymes in Arabidopsis roots. Plant $J$ 27:37-48.

Sawangrat K, Yamashita S, Tanaka A, Morishita M, Kusamori K, Katsumi H, Sakane T, and Yamamoto A (2019) Modulation of intestinal transport and absorption of topotecan, a BCRP substrate, by various pharmaceutical excipients and their inhibitory mechanisms of BCRP transporter. J Pharm Sci 108:1315-1325.

Schutte ME, Freidig AP, van de Sandt JJM, Alink GM, Rietjens IMCM, and Groten JP (2006) An in vitro and in silico study on the flavonoid-mediated modulation of the transport of 2-amino-1-methyl-6-phenylimidazo[4,5-b]pyridine (PhIP) through Caco-2 monolayers. Toxicol Appl Pharmacol 217:204-215.

Sengupta B and Sengupta PK (2003) Binding of quercetin with human serum albumin: a critical spectroscopic study. Biopolymers 72:427-434.

Shugarts S and Benet LZ (2009) The role of transporters in the pharmacokinetics of orally administered drugs. Pharm Res 26:2039-2054

Sjöstedt N, Holvikari K, Tammela P, and Kidron H (2017) Inhibition of breast cance resistance protein and multidrug resistance associated protein 2 by natural compounds and their derivatives. Mol Pharm 14:135-146. 
Studzian M, Bartosz G, and Pulaski L (2015) Endocytosis of ABCG2 drug transporter caused by binding of 5D3 antibody: trafficking mechanisms and intracellular fate. Biochim Biophys Acta 1853:1759-1771.

Studzian M and Pulaski L (2017) inventors. Method of studying Abcg2 protein activity and use of 2-aminoethyl diphenylborinate. European patent: EP3117212.

Szilagyi JT, Gorczyca L, Brinker A, Buckley B, Laskin JD, and Aleksunes LM (2019) Placental BCRP/ABCG2 transporter prevents fetal exposure to the estrogenic mycotoxin zearalenone. Toxicol Sci 168:394-404.

Taylor NMI, Manolaridis I, Jackson SM, Kowal J, Stahlberg H, and Locher KP (2017) Structure of the human multidrug transporter ABCG2. Nature 546:504-509.

Telbisz Á, Hegedüs C, Özvegy-Laczka C, Goda K, Várady G, Takáts Z, Szabó E, Sorrentino BP, Váradi A, and Sarkadi B (2012) Antibody binding shift assay for rapid screening of drug interactions with the human ABCG2 multidrug transporter. Eur J Pharm Sci 45:101-109.

Telbisz A, Müller M, Ozvegy-Laczka C, Homolya L, Szente L, Váradi A, and Sarkadi B (2007) Membrane cholesterol selectively modulates the activity of the human ABCG2 multidrug transporter. Biochim Biophys Acta 1768:2698-2713.

Williamson G, Kay CD, and Crozier A (2018) The Bioavailability, Transport, and Bioactivity of Dietary Flavonoids: A Review from a Historical Perspective. Comprehensive Reviews in Food Science and Food Safety 17:1054-1112.
Xiang D, Fan L, Hou X-L, Xiong W, Shi C-Y, Wang W-Q, and Fang J-G (2018) Uptake and transport mechanism of dihydromyricetin across human intestinal Caco-2 cells. J Food Sci 83:1941-1947.

Xie J-D, Huang Y, Chen D-T, Pan J-H, Bi B-T, Feng K-Y, Huang W, and Zeng W-A (2015) Fentanyl enhances hepatotoxicity of paclitaxel via inhibition of CYP3A4 and ABCB1 transport activity in mice. PLoS One 10:e0143701.

Yang Y, Hu Q, Fan Y, and Shen H (2008) Study on the binding of luteolin to bovine serum albumin. Spectrochim Acta A Mol Biomol Spectrosc 69:432-436.

Yang Z, Zhu W, Gao S, Yin T, Jiang W, and Hu M (2012) Breast cancer resistance protein (ABCG2) determines distribution of genistein phase II metabolites: reevaluation of the roles of ABCG2 in the disposition of genistein. Drug Metab Dispos 40:1883-1893.

Ye Q, Liu K, Shen Q, Li Q, Hao J, Han F, and Jiang R-W (2019) Reversal of multidrug resistance in cancer by multi-functional flavonoids. Front Oncol 9:487.

Address correspondence to: Lukasz Pulaski, Institute of Medical Biology, Polish Academy of Sciences, Lodowa 106, 93-232 Lodz, Poland. E-mail: lpulaski@uni.lodz.pl 